\title{
Diagnostic Accuracy of Electrographic Seizure Detection by Neurophysiologists and Non-Neurophysiologists in the Adult ICU Using a Panel of Quantitative EEG Trends
}

\author{
Christa B. Swisher, * Corey R. White, * Brian E. Mace, * Keith E. Dombrowski, * Aatif M. Husain, *广 \\ Bradley J. Kolls, ${ }^{*}$ Rodney R. Radtke, ${ }^{*}+$ Tung T. Tran, ${ }^{* \dagger}$ and Saurabh R. Sinha*†
}

Purpose: To evaluate the sensitivity and specificity of a panel of quantitative EEG (qEEG) trends for seizure detection in adult intensive care unit (ICU) patients when reviewed by neurophysiologists and non-neurophysiologists. Methods: One hour qEEG panels $(\mathrm{n}=180)$ were collected retrospectively from 45 ICU patients and were distributed to 5 neurophysiologists, 7 EEG technologists, and 5 Neuroscience ICU nurses for evaluation of seizures. Each panel consisted of the following qEEG tools, displayed separately for left and right hemisphere electrodes: rhythmicity spectrogram (rhythmic run detection and display; Persyst Inc), color density spectral array, EEG asymmetry index, and amplitude integrated EEG. The reviewers did not have access to the raw EEG data.

Results: For the reviewer's ability to detect the presence of seizures on qEEG panels when compared with the gold standard of independent raw EEG review, the sensitivities and specificities are as follows: neurophysiologists 0.87 and 0.61 , EEG technologists 0.80 and 0.80 , and Neuroscience ICU nurses 0.87 and 0.61 , respectively. There was no statistical difference among the three groups regarding sensitivity.

Conclusions: Quantitative EEG display panels are a promising tool to aid detection of seizures by non-neurophysiologists as well as by neurophysiologists. However, even when used as a panel, qEEG trends do not appear to be adequate as the sole method for reviewing continuous EEG data.

Key Words: Quantitative EEG, Continuous EEG, ICU EEG, Nonconvulsive seizures.

( Clin Neurophysiol 2015;32: 324-330)

From the *Department of Neurology, Duke University Medical Center, Durham, North Carolina, U.S.A.; and †Neurodiagnostic Center, Veterans Affairs Medical Center, Durham, North Carolina, U.S.A.

S. R. Sinha has received research support from UCB Pharma, Cyberonics, Upsher-Smith Laboratories, and Schwarz Pharma. He has received honoraria for lectures from UCB Pharma, Lundbeck and Cyberonics. He has provided consultation to Pfizer, Accorda, Upsher-Smith Laboratories, and Lundbeck. K. E. Dombrowski has received research support from Jordan Neuroscience, Optima Neuroscience, Sage Therapeutics, UCB Pharma, and Sanofi Aventis. A. M. Husain has received research support from UCB Pharma, Pfizer, Upsher-Smith Laboratories. He has received honoraria for lectures from UCB Pharma, Jazz Pharma. He has provided consultation to UCB Pharma, Upsher-Smith Laboratories, Jazz Pharma. He has received royalties from Demos Publishers for two books he has edited. B. J. Kolls has received research funding from Jordan Neuroscience, UCB Pharma, Optima Neuroscience, Medtronics and BrainScope Company, Inc. R. R. Radtke has received honoraria for lectures from UCB Pharma. He has provided consultation to UCB Pharma, Sunovian, Lundbeck, Easai, Supernus, Upsher-Smith, and Acorda. T. Tran has received honoraria for lectures from UCB Pharma.

Presented in part at the American Epilepsy Society Annual Meeting. Washington D.C., December 2013 and at Neurocritical Care Society Meeting, Philadelphia, PA, October 2013.

Address correspondence and reprint requests to Saurabh R. Sinha, MD, PhD, 295 Hanes House, 330 Trent Drive, Box 102350 Duke University Medical Center, Durham, NC 27710, U.S.A.; e-mail: saurabh.sinha@duke.edu.

Copyright $\odot 2015$ by the American Clinical Neurophysiology Society

ISSN: 0736-0258/15/3204-0324
Nonconvulsive seizures (NCS) and nonconvulsive status epilepticus occur frequently in patients admitted to the neurointensive care unit (ICU). Studies have found the incidence of NCS in different patient populations in medical or neurologic ICUs to be $8 \%$ to 37\% (Claassen et al., 2004; Jordan, 1995; Oddo et al., 2009; Pandian et al., 2004; Privitera et al., 1994; Towne et al., 2000). Because of the increased awareness of NCS and nonconvulsive status epilepticus in patients admitted to the Neuro-ICU, the utilization of continuous EEG (cEEG) monitoring has increased rapidly.

Quantitative EEG (qEEG) is a potential tool that can aid in the interpretation of the large volumes of EEG data generated by cEEG monitoring. Although commercial EEG software increasingly includes qEEG tools, the evidence for its diagnostic accuracy is sparse. Studies in the adult and pediatric population evaluating individual qEEG trends (envelope trend, amplitude integrated EEG [aEEG], or color density spectral array [CDSA]) derived from full-channel EEG montages report sensitivities for seizure detection from $41 \%$ to $89 \%$ (Abend et al., 2008; Ackman et al., 2011; Stewart et al., 2010; Williamson et al., 2014).

Approximately, $20 \%$ of seizures were missed using both CDSA and aEEG displays (Stewart et al., 2010). Amplitude integrated EEG has also been applied extensively to seizure detection in neonates (Tao and Mathur, 2010). Envelope trend analysis has also been shown to be fairly sensitive (88\%) for detecting longer seizures ( $\sim 1$ minute or longer) but performed less well for brief seizures (sensitivity of $40 \%$ ) and for slowly evolving seizures (sensitivity of 20\%) (Abend et al., 2008).

The majority of previous studies evaluated the use of a single qEEG trend. One study in the pediatric population detected an improvement in seizure detection when CDSA was combined with envelope trend analysis compared with envelope trend analysis alone (sensitivity of $66 \%$ vs. $50 \%$, respectively), but this benefit was only seen with experienced readers, not inexperienced readers (Ackman et al., 2011). Similarly, it has been our observation that using a panel of qEEG trends seems to enhance seizure detection compared with using a single type of trend. There have been no studies in the adult population assessing the sensitivity and specificity of qEEG graphical displays as a whole, derived from full-montage EEGs, in identifying seizures in adult ICU patients. The aim of this study was to evaluate the sensitivity and specificity of panels of qEEG trends for seizure detection in adult patients in the ICU when reviewed by neurophysiologists, EEG technologists, and Neuroscience ICU nurses.

\section{METHODS}

This study was approved by the Duke Institutional Review Board; consent for participation in the study was obtained from the 
neurophysiologists, technologists, and nurses. Thirty patients admitted to any of the Duke Intensive Care Units between 2010 and 2012 who had NCS identified on cEEG monitoring were identified retrospectively in a consecutive fashion from the Duke EEG database. Patients with one or more seizures in a 4-hour period were selected. Patients with nonconvulsive status epilepticus were not included in the study. Nonconvulsive status epilepticus was defined as continuous electrographic seizure activity lasting $>30$ minutes. The control group consisted of 15 patients who did not have NCS identified on cEEG monitoring. Clinical information for each patient was collected from the hospital electronic medical record.

All EEGs were collected with electrodes placed according to the International 10/20 electrode placement system. In addition, all cEEG recordings in the ICU were subjected to the qEEG tools built into the Magic Marker software (Persyst Development Corporation, Prescott, AZ). The corresponding raw EEG segments were reviewed independently by the study authors (C.B.S. and S.R.S.) to identify seizures. Electrographic seizures were identified using published criteria (Chong and Hirsch, 2005). Each 1-hour segment of raw EEG was placed in the following categories: no seizures, 1 to 2 seizures, 3 to 5 seizures, 6 to 10 seizures, or $>10$ seizures. For some records, there was a discrepancy in categorization of seizure number. In this case, the two authors (C.B.S. and S.R.S.) reviewed the studies together and established a consensus.

In addition, the average seizure duration, the spatial extent of the seizures (focal, defined as $\leq 4$ electrodes involved, hemispheric, defined as unilateral but $>4$ electrodes involved, or generalized/ bilateral), and primary background EEG abnormality were determined by the author, C.R.S., from the corresponding raw EEG segment for each qEEG panel. The EEG background pattern was classified as periodic if it consisted of lateralized periodic discharges or generalized periodic discharges and nonperiodic if it consisted of diffuse slowing, focal slowing, burst suppression pattern, or lateralized spike-and-wave or sharp-and-wave discharges (Hirsch et al., 2013). We chose to classify burst suppression as a nonperiodic pattern because the burst suppression samples in the study contained very long periods of suppression as well as variable durations of suppression for each patient.

Four qEEG panels (1 hour each) for each patient were collected from the cEEG file and deidentified, resulting in a total of 180 qEEG panels. An example panel is shown in Fig. 1. Each panel consisted of the following qEEG tools (based on all standard electrodes from left and right hemispheres): rhythmicity spectrogram (rhythmic run detection and display; Persyst Inc, displayed for the left and right hemispheres; range 1-24 Hz, 3-seconds epochs with 1-second step size), CDSA for the left and right hemispheres, based on fast Fourier transform power spectrum with 1 -second windows, range $0-20 \mathrm{~Hz}$, EEG asymmetry index (displayed as both absolute and relative values; for relative, upward deflection is left, downward is right), and aEEG (displayed for the left and right hemispheres; time constant of 0.16 seconds with 4seconds epochs).

Color density spectral array displays time on the x-axis, frequency on the y-axis and the amplitude of the power spectrum are encoded in different colors. The rhythmicity spectrogram is a proprietary qEEG tool from Persyst Inc that highlights the frequency components of the CDSA that have the highest amplitude and rhythmicity at a given time point, allowing for the rhythmic activity of seizures to be emphasized. The absolute asymmetry index compares the difference in power at each pair of homologous electrodes, and the difference is represented as a positive deflection. The relative asymmetry index also calculates the difference in power between homologous electrodes but provides lateralization (upward deflection with more power on the right and downward deflection with more power on the left). Amplitude integrated EEG depicts filtered, rectified, and smoothed EEG amplitude on a compressed time scale (Sinha, 2013).

The randomized qEEG panels $(\mathrm{n}=180)$ were distributed to 5 neurophysiologists, 7 EEG technologists, and 5 Neuroscience ICU nurses. All reviewers analyzed all 180 qEEG panels. These reviewers were asked to determine the number of seizures present in the panel with the options being: no seizures, 1 to 2 seizures, 3 to 5 seizures, 6 to 10 seizures or $>10$ seizures. The reviewers did not have access to the corresponding raw EEG data. Before evaluating the qEEG panels, the reviewers were given a brief 15-minute training session on $\mathrm{qEEG}$ through a Powerpoint presentation. The training session consisted of a basic description of each of the qEEG trends used in the study, examples of seizures on qEEG panels and several slides contrasting episodes of artifact from seizures. In addition, the reviewers were asked which qEEG tool they thought that was most useful in identifying seizures (rhythmicity spectrogram, CDSA, asymmetry index, or aEEG). Reviewers had no knowledge of clinical data for any recording.

Standard test characteristics (sensitivity, specificity, positive predictive value, and negative predictive value) were calculated for each group for the ability to detect the presence or absence of seizures. The responses that included 1 to 2 seizures, 3 to 5 seizures, 6 to 10 seizures, and $>10$ seizures were grouped as seizures being present for analyses that assessed for the presence or absence of seizures. The sensitivities of all individual readers were grouped by reader type (neurophysiologist, EEG technologist, and Neuroscience ICU nurse). These three groups were then compared using analysis of variance and a post hoc analysis with Tukey correction for multiple comparisons. In addition, interrater reliability within each group was calculated using Fleiss Kappa. Contingency analysis was used to make comparisons of the mean diagnostic accuracy of the 3 groups as determined by: (1) Seizure duration with short $(<25$ th percentile) versus long $(>75$ th percentile) seizures and (2) Background EEG pattern (periodic vs. nonperiodic). Pearson $\chi^{2}$ statistic was calculated for each these analyses. For the comparison of reviewer accuracy based on seizure spatial extent (focal, generalized, or hemispheric/bilateral), an overall $\chi^{2}$ with post hoc pairwise comparisons were performed. A Bonferroni calculation was performed to correct for multiple comparisons. For all calculations, a $P$-value $<0.05$ was considered statistically significant. All analysis was performed in STATA 13 (STATA Corp, College Station, TX).

\section{RESULTS}

A Tables 1 and 2 summarize the patient and EEG characteristics of the 45 patients from which the 180 qEEG panels were collected (30 patients with NCS and 15 patients without). The median age was 62 years (range, 21-95 years) and $60 \%$ of the patients were male. The majority of patients $(n=36,80 \%)$ were located in the Neuroscience ICU. The rest were located in the Cardiology ICU $(n=4)$, Medical ICU $(n=3)$, Cardiothoracic Surgery ICU $(n=1)$, and the Surgical ICU $(n=1)$. The primary diagnosis varied, with hypoxic-ischemic encephalopathy, brain tumor, and ischemic stroke being the most common diagnoses. 

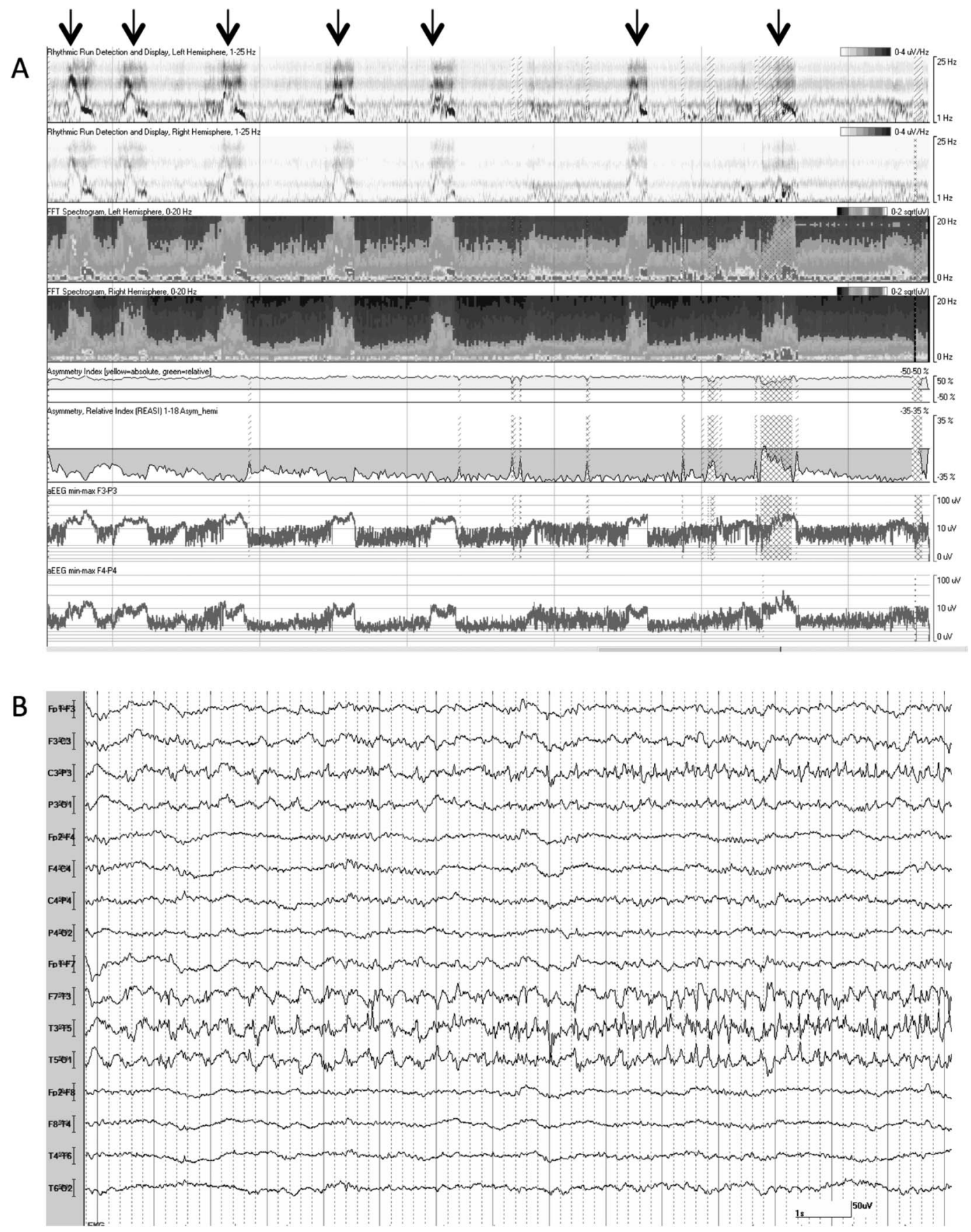

FIG. 1. Sample qEEG panel. Each qEEG panel was 1 hour duration and consisted of the following qEEG tools: rhythmicity spectrogram (displayed for the left and right hemispheres), color density spectral array (displayed for the left and right hemispheres), asymmetry index (displayed as both absolute and relative values), and aEEG (displayed for the left and right hemispheres). A, An example qEEG panel that was distributed is shown. Black arrows denote electrographic seizures. B, The corresponding raw EEG (16 seconds) for one of the seizures is shown. The background EEG consisted of left hemispheric lateralized periodic discharges occurring at a rate of 0.5 to $1 \mathrm{~Hz}$. Electrographic seizures consisted of evolving semi-rhythmic spike-and-wave discharges in the left temporal region with superimposed beta activity lasting 60 to 120 seconds followed by left hemispheric rhythmic delta activity. qEEG, quantitative EEG.

The majority of patients were initiated on cEEG monitoring for having a decreased level of consciousness $(n=29)$, followed by patients with observed clinical seizure activity and subsequent reduced level of consciousness $(n=14)$. Two patients were monitored for the presence of persistent focal neurologic deficits.

Of the 180 qEEG panels, there were 105 panels (58\%) that contained 1 or more seizures. The interrater reliability for 


\begin{tabular}{|c|c|c|}
\hline Total number of patients studied, $\mathrm{n}$ & \multicolumn{2}{|c|}{$45(60 \%$ male $)$} \\
\hline Patients with NCS & \multicolumn{2}{|c|}{30} \\
\hline Patients without NCS & \multicolumn{2}{|c|}{15} \\
\hline Age, median (range), years & \multicolumn{2}{|c|}{$62(21-95)$} \\
\hline \multicolumn{3}{|l|}{ Indication for cEEG monitoring, $\mathrm{n}$} \\
\hline Decreased level of consciousness & \multicolumn{2}{|c|}{29} \\
\hline Clinical seizure activity followed by & \multicolumn{2}{|c|}{14} \\
\hline decreased level of consciousness & & \\
\hline Focal neurologic deficits & \multicolumn{2}{|c|}{2} \\
\hline Primary diagnosis, $\mathrm{n}$ & Seizures & No seizures \\
\hline Brain tumor & 5 & 1 \\
\hline Ischemic stroke & 5 & 1 \\
\hline $\begin{array}{l}\text { History of seizures now with altered } \\
\text { mental status }\end{array}$ & 4 & 1 \\
\hline Subdural hematoma & 3 & 1 \\
\hline Traumatic brain injury & 3 & 1 \\
\hline Other & 3 & 1 \\
\hline Intracerebral hemorrhage & 3 & 0 \\
\hline Postoperative from brain tumor resection & 3 & 2 \\
\hline Hypoxic-ischemic encephalopathy & 1 & 6 \\
\hline Subarachnoid hemorrhage & 0 & 1 \\
\hline
\end{tabular}

neurophysiologists and EEG technologists was higher than the interrater reliability for Neuroscience ICU nurses $(\kappa=0.48,95 \%$ confidence interval [CI], $0.47-0.53$ for neurophysiologists, $\kappa=0.46$,

\section{TABLE 2. EEG Characteristics}

\begin{tabular}{|c|c|c|}
\hline $\begin{array}{l}\text { Number of 1-hour blocks } \\
\text { containing seizures, } \mathrm{n} \\
\text { Number of 1-hour blocks per } \\
\text { seizure quantification } \\
\text { category, } \mathrm{n}\end{array}$ & \multicolumn{2}{|c|}{105} \\
\hline $1-2$ seizures & \multicolumn{2}{|c|}{26} \\
\hline 3-5 seizures & \multicolumn{2}{|c|}{30} \\
\hline $6-10$ seizures & \multicolumn{2}{|c|}{32} \\
\hline$>10$ seizures & \multicolumn{2}{|c|}{17} \\
\hline $\begin{array}{l}\text { Seizure duration, median } \\
\text { (range), seconds }\end{array}$ & \multicolumn{2}{|c|}{$62(14-450)$} \\
\hline \multicolumn{3}{|l|}{ Seizure distribution, $\%$} \\
\hline Generalized or bilateral & \multicolumn{2}{|c|}{49} \\
\hline Hemispheric & \multicolumn{2}{|c|}{17} \\
\hline Focal & \multicolumn{2}{|c|}{34} \\
\hline $\begin{array}{l}\text { Baseline EEG background } \\
\text { primary abnormality } \\
\text { per 1-hour block, n }(\%)\end{array}$ & Seizures $(\mathrm{n}=105)$ & No seizures $(n=75)$ \\
\hline $\begin{array}{l}\text { Lateralized spike-and-wave } \\
\text { or sharp-and-wave discharges }\end{array}$ & $35(33)$ & $23(31)$ \\
\hline $\begin{array}{l}\text { Lateralized periodic } \\
\text { discharges* }\end{array}$ & $26(25)$ & $4(5)$ \\
\hline $\begin{array}{l}\text { Generalized periodic } \\
\text { discharges* }\end{array}$ & $4(4)$ & $8(11)$ \\
\hline Focal slowing & $22(21)$ & $10(13)$ \\
\hline Diffuse slowing & $4(4)$ & $24(32)$ \\
\hline Burst suppression pattern & $14(13)$ & $6(8)$ \\
\hline
\end{tabular}

*EEG patterns defined as periodic. All other EEG patterns were classified nonperiodic. Burst suppression was classified as a nonperiodic pattern because the burst suppression samples in the study contained very long periods of suppression as well as variable durations of suppression for each patient.
95\% CI, 0.43-0.49 for EEG technologists, and $\kappa=0.33,95 \% \mathrm{CI}$, 0.30-0.39 for Neuroscience ICU nurses).

For the reviewer's ability to detect the presence of seizures on qEEG panels when compared with the gold standard of independent raw EEG review, the sensitivities and specificities are as follows: neurophysiologists 0.87 and 0.61 , EEG technologists 0.80 and 0.80 , and Neuroscience ICU nurses 0.87 and 0.61 , respectively. The positive predictive value for a seizure was $76 \%$ for neurophysiologists, $85 \%$ for EEG technologists, and $76 \%$ for Neuroscience ICU nurses. The negative predictive value for a seizure was $77 \%$ for neurophysiologists, $74 \%$ for EEG technologists, and $77 \%$ for Neuroscience ICU nurses. The data are displayed in Tables 3 and 4 . There was no statistical difference in sensitivity between the three groups regarding the detection of the presence or absence of seizures. The false positive rate for all reviewers was $31 \%$ (neurophysiologists 39\%, EEG technologists 20\%, and Neuroscience ICU nurses 38\%).

All reviewers displayed a poorer performance when asked to identify the correct category for the number of seizures (all reviewers $51 \%$ correct, neurophysiologists $49 \%$ correct, EEG technologists $47 \%$ correct, and Neuroscience ICU nurses 55\% correct) with the categories being: no seizures, 1 to 2 seizures, 3 to 5 seizures, 6 to 10 seizures, or $>10$ seizures.

We then looked at whether certain characteristics of the EEG background or electrographic seizures significantly influenced sensitivity and specificity. When comparing short seizures $(<25$ th percentile, $<41$ seconds) with long seizures ( $>75$ th percentile, $>121$ seconds), there was a statistically significant difference in the neurophysiologist's and EEG technologist's ability to detect the presence of seizures in qEEG panels that contained, on average, longer seizures. Neuroscience ICU nurses were not more likely to detect long seizures than short seizures. Neurophysiologists and EEG technologists were statistically more likely to detect general$\mathrm{ized} /$ bilateral seizures and hemispheric seizures than focal seizures. Seizure spatial extent did not influence the accuracy of Neuroscience ICU nurses. Only the group of neurophysiologists were more likely to detect the presence or absence of seizures when the background EEG for the corresponding qEEG panel was periodic (84\% correct) versus nonperiodic ( $73 \%$ correct) $(P=0.004)$. The data are summarized in Table 5, which includes associated $P$ values.

When neurophysiologists correctly identified seizures, rhythmicity spectrogram was believed to be the most helpful trend, $57 \%$ of the time. Similarly, Neuroscience ICU nurses also most often relied on rhythmicity spectrogram as the most helpful trend (43\%). EEG technologists, however, relied mostly on aEEG (38\%). The data are displayed in Fig. 2.

\section{DISCUSSION}

This study shows that isolated review of a panel of commonly used qEEG trends demonstrates an overall sensitivity of $84 \%$ and specificity of $69 \%$ for all reviewer types for the detection of the presence of seizures. Despite the fact that neurophysiologists and EEG technologists had some previous experience with qEEG, Neuroscience ICU nurses had little to no experience with qEEG, and there was no statistical difference in the sensitivities among the three groups (neurophysiologists 0.87 , EEG technologists 0.80 , and Neuroscience ICU nurses 0.87 ). Therefore, qEEG remains a promising tool that can be used by Neuroscience ICU nurses and EEG technologists in addition to neurophysiologists to aid in the interpretation of large volumes of data generated by cEEG 
TABLE 3. Sensitivity, Specificity, Positive Predictive Value, and Negative Predictive Value Comparison Among Neurophysiologists, EEG Technologists, and Neuroscience Intensive Care Unit Nurses Using Quantitative EEG Panels for the Detection of Seizures

\begin{tabular}{lcccc}
\hline & Mean Sensitivity & Mean Specificity & Mean PPV & Mean NPV \\
\hline Neurophysiologists $(\mathrm{n}=5)$ & $87.4(84.3-90.1)$ & $61.1(55.9-66.0)$ & $75.9(72.3-79.2)$ & $77.6(72.4-82.3)$ \\
EEG technologists $(\mathrm{n}=7)$ & $79.7(76.6-82.5)$ & $79.8(76.2-83.1)$ & $84.7(81.7-87.2)$ & $73.7(69.9-77.3)$ \\
Neuro-ICU nurses $(\mathrm{n}=5)$ & $87.1(83.3-89.7)$ & $61.6(56.4-66.5)$ & $76.2(72.5-79.5)$ & $77.1(71.8-81.7)$ \\
Total $(\mathrm{n}=17)$ & $84.1(82.4-85.8)$ & $68.9(66.3-71.5)$ & $79.2(77.2-81.0)$ & $75.6(73.0-78.0)$ \\
\hline
\end{tabular}

ICU, intensive care unit; NPV, negative predictive value; PPV, positive predictive value.

monitoring. Further studies are needed to evaluate the ability of nonneurophysiologists to prospectively identify NCS with the use of qEEG panels running at the bedside.

Studies in the pediatric ICU population have shown that the sensitivity of individual qEEG trends (CDSA and aEEG), derived from limited channel cEEGs, are $22 \%$ to $80 \%$ (Evans et al., 2010; Pensirikul et al., 2013; Rennie et al., 2004; Shah et al., 2008; Shellhaas et al., 2007; Toet et al., 2002). Studies in the adult and pediatric population also evaluating individual qEEG trends (aEEG, CDSA, or envelope trend), but derived from full channel cEEGs, report higher sensitivities for seizure detection from $41 \%$ to $89 \%$ (Abend et al., 2008; Ackman et al., 2011; Stewart et al., 2010; Williamson et al., 2014). The higher sensitivities in these studies may be attributed to the fact that the qEEG data were derived from full-channel EEG montages rather than limited-channel EEG montages. These studies evaluated both experienced and inexperienced qEEG readers, possibly explaining the wide range of reported sensitivities.

When read by experienced neurophysiologists, a qEEG panel of trends generated from full-montage cEEGs in this study may have a slightly improved sensitivity as compared with previously reported trials evaluating single qEEG trends (aEEG, CDSA, or envelope trend) also generated from full-montage cEEGs read by neurophysiologists ( $87 \%$ vs. 50\%-83\%) (Abend et al., 2008; Ackman et al., 2011; Stewart et al., 2010). However, the use of a panel of qEEG trends may be most useful to nonexperienced readers as the sensitivity for EEG technicians and Neuroscience nurses in this study was 0.80 and 0.87 , respectively, whereas the sensitivity for nonexperienced readers (neurology residents) using envelope trend was previously reported to be $41 \%$ to $50 \%$ (Abend et al., 2008; Ackman et al., 2011). However, a direct comparison is difficult because these studies were performed in the neonatal and pediatric patient population. The difference in sensitivity of seizure detection may be a reflection of the characteristics of neonatal versus adult

TABLE 4. Sensitivity Comparison Among Neurophysiologists, EEG Technologists and Neuroscience Intensive Care Unit Nurses for the Detection of Seizures on Quantitative EEG Panels

\begin{tabular}{llr}
\hline & Mean Difference & $\boldsymbol{P}$ \\
\hline Neurophysiologists vs. EEG technologists & $7.73(-6.39$ to 21.9$)$ & 0.35 \\
Neurophysiologists vs. nurses & $0.38(-14.9$ to 15.6$)$ & 1.00 \\
Nurses vs. EEG technologists & $7.35(-6.8$ to 21.5$)$ & 0.39 \\
\hline \multicolumn{2}{c}{ Data are number identified (95\% confidence interval). Statistical analysis was } \\
performed by analysis of variance with post hoc analysis with Tukey correction for \\
multiple comparisons.
\end{tabular}

seizures. The study by Ackman et al. detected an improved sensitivity for experienced readers only with envelope trend analysis and CDSA when compared with envelope trend analysis alone. Although the sample size was low in this study $(n=6)$, this further supports the hypothesis that a panel of qEEG trends may be superior to individual qEEG trends for seizure detection.

There has been one recent study evaluating the use of qEEG in the adult population. A study by Williamson et al. assessed the use of CDSA by non-neurophysiologists to identify seizures in adult patients and found a seizure detection rate of $89 \%$. However, by trial design, there was a high false positive rate (for every seizure detected

TABLE 5. Seizure Characteristics and Percentage of Correct Identification on Quantitative EEG Panels Among All Reviewers and the Three Individual Groups (Neurophysiologists, EEG Technologists, and Neuroscience Intensive Care Unit Nurses)

\begin{tabular}{|c|c|c|c|}
\hline & \multicolumn{3}{|c|}{ Seizure Duration (\% Correct) } \\
\hline & Short* & Long* & $P$ \\
\hline All reviewers & 78.2 & 85.7 & $<\mathbf{0 . 0 1}$ \\
\hline Neurophysiologists & 77.0 & 87.1 & 0.03 \\
\hline EEG technologists & 74.6 & 83.2 & 0.04 \\
\hline \multirow[t]{3}{*}{ Neuro-ICU nurses } & 84.4 & 87.9 & 0.41 \\
\hline & \multicolumn{3}{|c|}{ Seizure Spatial Extent (\% Correct) } \\
\hline & Hemispheric & Generalized/bilateral & Focal \\
\hline All reviewers & $87.6^{\mathrm{A}}$ & $91.8^{\mathrm{A}}$ & $78.6^{\mathrm{B}}$ \\
\hline Neurophysiologists & $90.6^{\mathrm{A}}$ & $94.4^{\mathrm{A}}$ & $82.0^{\mathrm{B}}$ \\
\hline EEG technologists & $84.1^{\mathrm{A}}$ & $92.1^{\mathrm{A}}$ & $71.7^{\mathrm{B}}$ \\
\hline \multirow[t]{3}{*}{ Neuro-ICU nurses } & $89.4^{\mathrm{A}}$ & $88.9^{\mathrm{A}}$ & $84.7^{\mathrm{A}}$ \\
\hline & \multicolumn{3}{|c|}{ EEG Background (\% Correct) } \\
\hline & Periodic & Nonperiodic & $P$ \\
\hline All reviewers & 83.0 & 76.8 & $<\mathbf{0 . 0 1}$ \\
\hline Neurophysiologists & 84.0 & 73.0 & $<\mathbf{0 . 0 1}$ \\
\hline EEG technologists & 84.7 & 80.3 & 0.08 \\
\hline Neuro-ICU nurses & 80.0 & 75.0 & 0.13 \\
\hline
\end{tabular}

Short seizures were defined as $<25$ th percentile, $<41$ seconds, and long seizures were defined as $>75$ th percentile, $>121$ seconds. Contingency analysis was used to make comparisons of the mean diagnostic accuracy of the three groups as determined by: (1) Seizure duration with short $(<25$ th percentile) versus long $(>75$ th percentile) seizures; (2) Background EEG pattern (periodic vs. nonperiodic). The $\chi^{2}$ statistic was calculated for each these analyses. For the comparison of reviewer accuracy based on seizure spatial extent, an overall $\chi^{2}$ with post hoc pairwise comparisons were performed. Bolded values represent statistically significant $P$ values. Treatments with the same label (A or B) are statistically equivalent. Treatments with different labels differ with $P<$ 0.05 after Bonferroni correction for multiple comparisons.

ICU, intensive care unit. 


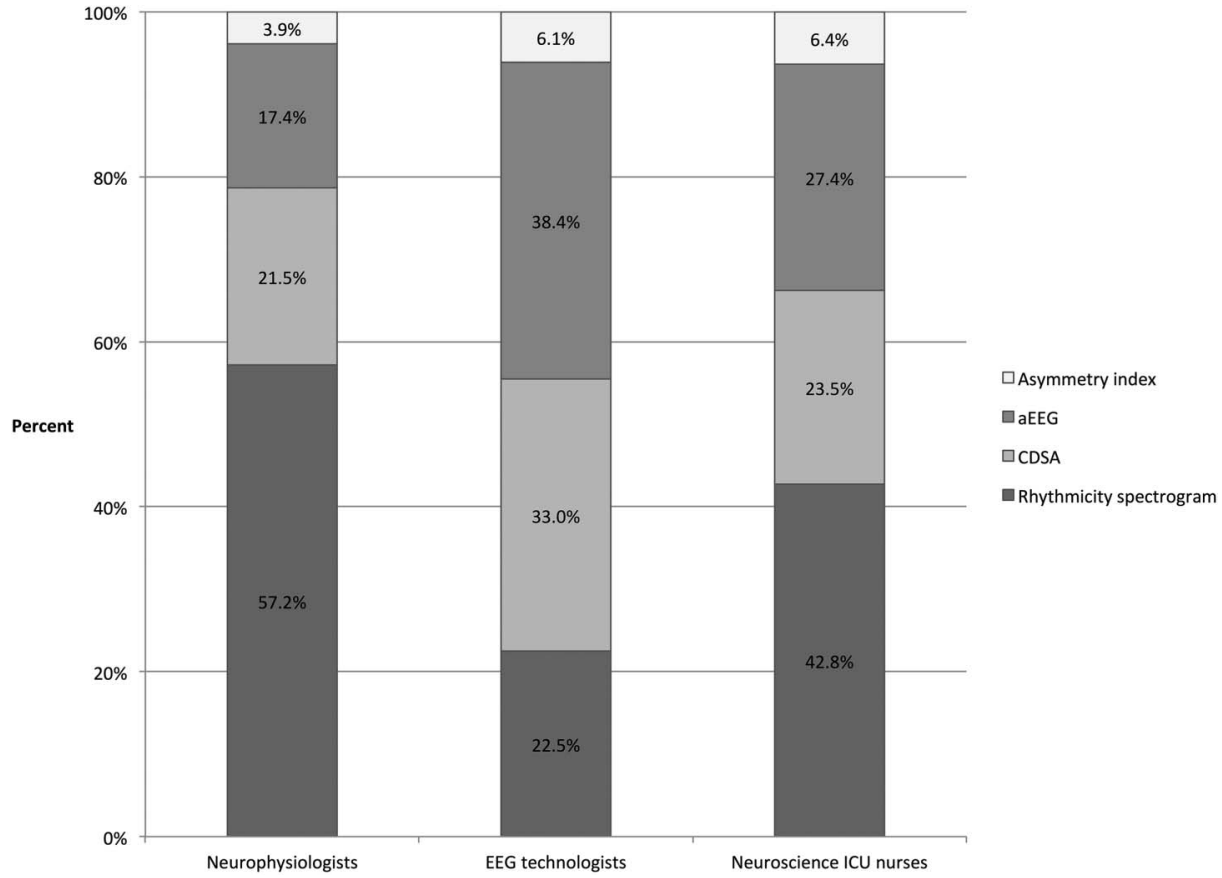

FIG. 2. Quantitative EEG trend importance as determined by neurophysiologists, EEG technologists, and Neuroscience ICU nurses. Each reviewer was asked to select the most helpful qEEG trend (rhythmicity spectrogram, CDSA, aEEG, and asymmetry index) for each qEEG panel. The data in each column represent the cumulative responses for each reviewer type for correct identification of seizures in the 105 qEEG panels that contained seizures. aEEG, amplitude integrated EEG; CDSA, color density spectral array; $I C U$, intensive care unit; qEEG, quantitative EEG. there was 13.8 segments that were marked by the readers that did not contain seizures) (Williamson et al., 2014). The specificity was not reported in this study. The goal was for the inexperienced reader (neurology residents) to identify any significant changes in the CDSA rather than to identify seizures only. Their results support the prospect of having non-neurophysiologists identify periods of concern on the qEEG. The study design by Williamson et al. is significantly different than the design of this study (marking areas of concern vs. marking suspected seizures), therefore not allowing for direct comparisons.

A larger concern was that even with an acceptable sensitivity and specificity in this study, there was a false negative rate of $16 \%$. This is similar to a previously published report on the use of CDSA and aEEG for seizure detection in the neonatal ICU where a missed seizure rate of $20 \%$ was reported (Stewart et al., 2010). However, we hypothesize that the false negative rate in this study would be improved if the reviewers were given subsequent qEEG panels in series for each patient, which may reflect actual clinical practice more closely. The study by Stewart et al. demonstrated that missed seizures were more likely to be of short duration ( $<1$ minute), seizures that were focal in spatial extent and seizures that occurred in the presence of abundant interictal discharges (Stewart et al., 2010). Similarly, the current results also showed that, overall, there was poorer seizure detection with focal seizures when compared with hemispheric or generalized/bilateral seizures. In this study, neurophysiologists and EEG technologists were less likely to correctly detect short $(<25$ th percentile) versus long $(>75$ th percentile) seizures; however, this was not the case with Neuroscience ICU nurses. Another factor that might impact sensitivity is the presence of noise/artifact. Presumably a seizure would be harder to detect in the presence of a noisy background EEG. However, this is difficult to quantify, and we did not specifically evaluate it in this study.

There is a concern that a false positive rate of $31 \%$ (for all reviewer types) may lead to unnecessary treatment. Presumably, many of these false positives are related to state change or artifact. If qEEG trends are used as the sole method of review, the current data show that an unacceptably high rate of false alarms would occur. However, if qEEG trends are used in conjunction with raw EEG by those that have been trained in reading EEGs, the false positives would presumably be recognized and have less of an impact on clinical decision making. Therefore, the low specificity evident from using qEEG should be balanced with selective review of files that showed evidence of seizure activity. Given that this is a retrospective study, it remains to be determined whether qEEG should have a role in prospective EEG analysis by non-neurophysiologists. Theoretically, there may be an improvement in specificity and sensitivity with additional qEEG training and experience that would reduce the number of false alarms.

Despite an improvement in seizure detection with the use of a panel of qEEG trends, the ability to quantify the number of seizures was poor. Correct identification of specific categories (no seizures, 1-2 seizures, $3-5$ seizures, $6-10$ seizures, or $>10$ seizures) occurred only $51 \%$ of the time. In practice, qEEG would rarely be used in isolation, thus identification of the precise number of seizures may not be essential. If seizures are present, this would presumably lead to a more detailed review of the raw EEG and more precise quantification of the seizure number, if needed. Furthermore, the clinical relevance of the precise number of seizures is uncertain. In practice, knowing present versus absent and many versus few may be adequate to determine the patient's response to therapy.

Given the poor performance on attempts to quantify numbers of seizures in conjunction with a relatively high false positive rate, a more practical approach for the use of qEEG by nonneurophysiologists may involve identification of recurrent NCS after initial seizure identification on raw EEG. If the seizures can be clearly demonstrated in the qEEG trend, then it may be highly valuable and accurate at quantification in this specific setting, although this needs further study.

Although the reviewers had varying experience with the use of qEEG, the interrater reliabilities were similar among the three groups. There was moderate agreement among neurophysiologists and EEG technicians ( $\kappa=0.48$ and 0.46 , respectively) and fair 
agreement among Neuroscience ICU nurses $(\kappa=0.33)$. Given that Neuroscience ICU nurses have little to no experience with qEEG at our institution, it is expected that there would be less agreement among them. Since qEEG is not used on a regular clinical basis at our institution, we suspect that there would be improved agreement with more in-depth training as well as the implementation increased clinical usage of qEEG.

There are several potential limitations to this study. In this study, seizures were present in $58 \%$ of the qEEG panels. Since seizures are present in $17 \%$ to $20 \%$ of all patients undergoing cEEG monitoring at our institution (unpublished data), the reviewers may have been biased toward detecting seizures in this enriched dataset. The sensitivity and specificity may have been significantly decreased if only $17 \%$ to $20 \%$ of the qEEG panels contained seizures. Another potential limitation of the study was that the primary diagnosis differed significantly between the two groups. Hypoxic-ischemic encephalopathy was the most common diagnosis among patients with seizures on cEEG, whereas brain tumor was the most common diagnosis among patients without seizures. This is likely a reflection of the seizure likelihood of the various patient populations at our institution. However, the primary goal of the study was to evaluate the diagnostic accuracy of seizure detection by various $\mathrm{qEEG}$ readers, making it unlikely that the underlying diagnosis would affect this outcome.

The reviewers did not have access to the corresponding raw EEG segments. This was done to evaluate the sensitivity and specificity of a panel of qEEG trends alone for the detection of seizures. Typically, qEEG trends are used in conjunction with the raw EEG. If reviewers had access to the underlying raw EEG, an improvement in the sensitivity and specificity would be expected. However, this study more closely emulates the situation when nonneurophysiologists (e.g., nurses and other ICU staff) are asked to use qEEG trends to detect seizures at the bedside. This would potentially allow earlier detection of seizures compared with intermittent review of raw EEG by a neurophysiologist. Additionally, this study did not evaluate other commonly used qEEG trends (e.g., envelope trend) aside from rhythmicity spectrogram, CDSA, aEEG, and asymmetry index. This study did not evaluate how differences in the display of the qEEG panel (e.g., time per screen) would affect the reviewer's performances. An additional potential limitation to this study involves the identification of the number of seizures. Given that there were typically numerous seizures in an epoch, it would be difficult to come to an agreement about the precise number of seizures. We believed that categorization of the number of seizures was more reproducible and more clinically relevant.

Quantitative EEG display panels remain a promising tool to aid detection of seizures by neurophysiologists and nonneurophysiologists. However, even when used as a panel, qEEG trends do not seem to be sufficient as the sole method for reviewing cEEG data. Additional training for users, additional experience in the use of qEEG trends, use of other qEEG trends and simultaneous availability of raw EEG with qEEG trends might be helpful. In addition, using qEEG trends as a means for non-neurophysiologists may still be a reasonable means to identify periods of concern during live recording.

\section{ACKNOWLEDGMENTS}

The authors would like to acknowledge the Donald B. Sanders Neurology Fellows Research Grant for research funding support as well as Rizwana Rehman and Jonathan C. Riboh, MD for assistance with statistical analysis.

\section{REFERENCES}

Abend NS, Dlugos D, Herman S. Neonatal seizure detection using multichannel display of envelope trend. Epilepsia 2008;49:349-352.

Ackman CI, Micic V, Thompson A, Riviello JJ. Seizure detection using digital trend analysis: factors affecting utility. Epilepsy Res 2011;93:66-72.

Chong DJ, Hirsch LJ. Which EEG patterns warrant treatment in the critically ill? Reviewing the evidence for treatment of periodic epileptiform discharges and related patterns. J Clin Neurophysiol 2005;22:79-91.

Claassen J, Mayer SA, Kowalski RG, et al. Detection of electrographic seizures with continuous EEG monitoring in critically ill patients. Neurology 2004;62:1743-1748.

Evans E, Koh S, Lerner J, et al. Accuracy of amplitude integrated EEG in a neonatal cohort. Arch Dis Child Fetal Neonatal Ed 2010;95:F169-F173.

Hirsch L, LaRoche S, Gaspard N, et al. American Clinical Neurophysiology Society's Standardized Critical Care EEG Terminology: 2012 version. J Clin Neurophysiol 2013;30:1-27.

Jordan KG. Neuropysiologic monitoring in the neuroscience intensive-care Unit. Neurol Clin 1995;13:579-626.

Oddo M, Carrera E, Claassen J, et al. Continuous electroencephalography in the medical intensive care unit. Crit Care Med 2009;37:2051-2056.

Pandian JD, Cascino GD, So EL, et al. Digital video-electroencephalographic monitoring in the neurological-neurosurgical intensive care unit - clinical features and outcome. Arch Neurol-chicago 2004;61:1090-1094.

Pensirikul AD, Beslow LA, Kessler SK, et al. Density spectral array for seizure identification in critically ill Children. J Clin Neurophysiol 2013;30:371-375.

Privitera M, Hoffman M, Moore JL, Jester D. Eeg detection of Nontonic-Clonic status epilepticus in patients with Altered consciousness. Epilepsy Res 1994;18:155-166.

Rennie JM, Chorley G, Boylan GB, et al. Non-expert use of the cerebral function monitor for neonatal seizure detection. Arch Dis Child Fetal Neonatal Ed 2004;89:F37-F40.

Shah DK, Mackay MT, Lavery S, et al. Accuracy of bedside electroencephalographic monitoring in comparison with simultaneous continuous conventional electroencephalography for seizure detection in term infants. Pediatrics 2008;121:1146-1154.

Shellhaas RA, Soaita AI, Clancy RR. Sensitivity of amplitude-integrated electroencephalography for neonatal seizure detection. Pediatrics 2007;120:770-777.

Sinha SR. Quantitative EEG principles. In: LaRoche SM, eds. Handbook of ICU EEG monitoring. New York: Demos Medical Publishing, 2013:221-227.

Stewart CP, Otsubo H, Ochi A, et al. Seizure identification in the ICU using quantitative EEG displays. Neurology 2010;75:1501-1508.

Tao JD, Mathur AM. Using amplitude-integrated EEG in neonatal intensive care. $J$ Perinatol 2010;30(suppl):S73-S81.

Towne AR, Waterhouse EJ, Garnett L. Prevalence of nonconvulsive status epilepticus in comatose patients-reply from the authors. Neurology 2000;55:1422-1423.

Toet MC, van der Meij W, de Vries LS, et al. Comparison between simultaneously recorded amplitude integrated electroencephalogram (cerebral function monitor) and standard electroencephalogram in neonates. Pediatrics 2002;109:772-779.

Williamson CA, Wahlster S, Shafi MM, Westover MB. Sensitivity of compressed spectral arrays for detecting seizures in acutely ill adults. Neurocrit Care 2014;20:32-39. 